\title{
PARTIALLY INFORMED INVESTORS: HEDGING IN AN INCOMPLETE MARKET WITH DEFAULT
}

\author{
P. TARDELLI,* University of L'Aquila
}

\begin{abstract}
In a defaultable market, an investor trades having only partial information about the behavior of the market. Taking into account the intraday stock movements, the risky asset prices are modelled by marked point processes. Their dynamics depend on an unobservable process, representing the amount of news reaching the market. This is a marked point process, which may have common jump times with the risky asset price processes. The problem of hedging a defaultable claim is studied. In order to discuss all these topics, in this paper we examine stochastic control problems using backward stochastic differential equations (BSDEs) and filtering techniques. The goal of this paper is to construct a sequence of functions converging to the value function, each of these is the unique solution of a suitable BSDE.
\end{abstract}

Keywords: Optimal investment; exponential utility; default time; dynamic programming; backward stochastic differential equation; filtering

2010 Mathematics Subject Classification: Primary 49L20

Secondary 93E11; 93E03

\section{Introduction}

This paper deals with the problem of hedging a defaultable claim. On this topic a large literature can be found; see, e.g. Bielecki et al. [1], Jeanblanc et al. [13], and the references therein. A defaultable contingent claim maturing at time $T$ consists of a triplet $(Z, \tilde{Z}, \tau)$. The random variable $\tau$ models the time of default. The random variable $Z$ is the payoff received by the owner of the claim at $T$ if the default does not occur before maturity. The so-called recovery process $\tilde{Z}_{t}$ is such that $\tilde{Z}_{\tau}$ is the payoff received at the time of default if $\tau \leq T$.

Most of the standard literature in finance is based on the assumption that the prices of the underlying assets follow a diffusion behavior. However, empirical evidence from various studies show that such models are inadequate for different reasons, e.g. the mispricing that they might induce; see Runggaldier [18]. As already observed in Geradi and Tardelli [10], on very small time scales actual prices do not really change continuously over time, but rather at discrete random points in time in reaction to trades and/or to significant new information. Moreover, the advent of intraday information on financial asset price quotes and the increasing number of studies on market microstructure leads us to describe the prices as processes that are piecewise constant and jump at irregularly spaced random times in reaction to trades or to significant new information. Therefore, pure jump processes may be more suitable for modelling the observed price or quantities related to the price; see, e.g. Prigent [17].

Received 10 July 2013; revision received 30 June 2014.

* Postal address: Department of Industrial and Information Engineering and Economics, University of L'Aquila, 67100, Italy. Email address: paola.tardelli@univaq.it 
In this paper the prices are studied by introducing a model similar to that presented by Tardelli [20], [21], and Gerardi and Tardelli [10]. In particular, the dynamics of the underlying asset price $S$ is described by a pure jump process driven by two point processes and by the default indicating process. Many authors (e.g. Zariphopoulou [22], even if in a continuous behavior) claim that it is sensible to assume that the price dynamics depend on an exogenous process. According to this idea, the dynamics of the price is assumed to depend on a pure jump process $X$. Moreover, $S$ and $X$ may have common jump times and $X$ is unobservable by the market agents.

Taking into account the presence of the unobservable process $X$, in this paper we consider a market with an investor who does not have complete information about the dynamics of the market. He/she just observes the price behavior and the default time. The common way to solve this problem is to use filtering theory so as to reduce a stochastic control problem with partial information to one with complete information. Thus, a technique of projection is needed that leads to a filtering problem with marked point process observations. This latter topic is discussed in a way similar to that used in Ceci [5] and Tardelli [20].

Due to the presence of a pure jump price process, the market is incomplete. In such cases, there is always some residual risk and so one may want to choose a strategy so as to minimize a criterion related to this risk. This means that perfect replication is not possible and a suitable alternative approach must be found. Here, the procedure consists of maximizing the mean value of a utility function from the terminal wealth and choosing an exponential utility function; see, e.g. Hu et al. [11] and Mania and Schweizer [16]. The problem becomes a stochastic control problem and to deal with it many techniques can be used ranging from the dynamic programming method, Hamilton-Jacobi-Bellman partial differential equations, and backward stochastic differential equations (BSDEs), to duality methods. The approach followed here is related to dynamic programming, as in Tardelli [20], [21], and it allows us to characterize the value function as the largest solution to a suitable BSDE.

The main contribution of this paper is in Section 4, which is devoted to the construction of a sequence of functions converging to the value function. Each of these functions is the unique solution of suitable BSDEs, not only the largest one, as in a large part of the existing literature.

Let us note that many techniques used in this paper are inspired by the procedure followed by Lim and Quenez [15], even if a diffusive model is investigated in that paper.

\section{The market model}

Let us consider an economy defined on a complete real-world probability space, $(\Omega, \mathcal{F}, \mathbb{P})$ for a finite time $\operatorname{span}[0, T], T \in(0,+\infty)$, equipped with $\left\{\mathcal{F}_{t}\right\}_{t \in[0, T]}$, a filtration satisfying the usual conditions and on which all stochastic processes are defined. In this setup, let us consider a financial market in which two assets are traded: one risk-free asset, whose price process is assumed for simplicity to be equal to 1 at any date, and one risky asset. The price $S$ of the risky asset, discounted with respect to the price of the bond, is a process evolving according to the following model:

$$
S_{t}=S_{0} \exp \left\{Y_{t}\right\} \quad \text { with } S_{0} \in \mathbb{R}^{+} .
$$

The marked point process $Y$, called the log return price, is assumed to be nonexplosive $\mathbb{R}$-valued with initial condition $Y_{0}=0$. The dynamics of $Y$ depend on another exogenous marked point process $X$, which, in general, is not directly observable by the agents and which represents the amount of news reaching the market. Let us assume that $X$ is nonexplosive, taking values in $\mathbb{R}$ with initial condition $X_{0}=0$. 
Let $\tau$ be a positive random variable which models a default time. This default can appear at any time, i.e. $\mathbb{P}(\tau>t)>0$ for any $t \in[0, T]$. For $t \geq 0$, let the default indicating process be defined by $D_{t}:=\mathbf{1}_{\{\tau \leq t\}}$ and, consequently, let the filtration generated be $\mathscr{D}:=\left\{\mathscr{D}_{t}\right\}_{t \in[0, T]}$ with $\mathscr{D}_{t}:=\sigma\left\{D_{s}, 0 \leq s \leq t\right\}$. Let $\mathcal{O}:=\left\{\mathcal{O}_{t}\right\}_{t \in[0, T]}$ and $g_{s}:=\left\{g_{t}\right\}_{t \in[0, T]}$ be the filtrations defined by

$$
\mathcal{O}_{t}:=\sigma\left\{Y_{s}, D_{s}, 0 \leq s \leq t\right\}, \quad g_{t}:=\sigma\left\{X_{s}, Y_{s}, D_{s}, 0 \leq s \leq t\right\} .
$$

All the filtrations satisfy the usual hypotheses of completeness and right-continuity.

Let $\tau$ admit a positive $g_{t}$-predictable intensity. This means that there exists $\left\{\gamma_{t}\right\}_{t \geq 0}$ bounded, nonnegative, $g_{t}$-predictable process such that

$$
0<\gamma_{t} \leq \Lambda
$$

for a positive suitable constant $\Lambda \in \mathbb{R}^{+}$and, setting $\lambda_{s}^{3}:=\left(1-D_{s-}\right) \gamma_{s}$,

$$
M_{t}^{3}:=D_{t}-\int_{0}^{t \wedge \tau} \gamma_{s} \mathrm{~d} s=D_{t}-\int_{0}^{t} \lambda_{s}^{3} \mathrm{~d} s \quad \text { for } t \geq 0
$$

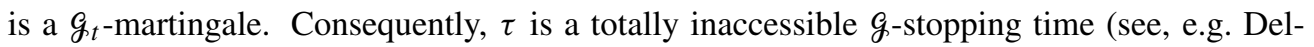
lacherie and Meyer [6, Section VI78]).

Let $N^{0}, N^{1}$, and $N^{2}$ be nonexplosive counting processes defined as

$$
\begin{gathered}
N_{t}^{0}=\sum_{s \leq t} \mathbf{1}_{\left\{X_{s}-X_{s-} \neq 0\right\}} \mathbf{1}_{\left\{Y_{s}-Y_{s-}=0\right\}}\left(1-D_{s}\right), \\
N_{t}^{1}=\sum_{s \leq t} \mathbf{1}_{\left\{Y_{s}-Y_{s-}>0\right\}}\left(1-D_{s}\right), \quad N_{t}^{2}=\sum_{s \leq t} \mathbf{1}_{\left\{Y_{s}-Y_{s-}<0\right\}}\left(1-D_{s}\right) .
\end{gathered}
$$

For $i=0,1,2$, let us assume that the process $N^{i}$ admits a $g_{t}$-predictable intensity. This means that there exist $\lambda_{t}^{0}, \lambda_{t}^{1}$, and $\lambda_{t}^{2}$ bounded nonnegative $g_{t}$-adapted processes such that

$$
0<\lambda_{t}^{i} \leq \Lambda
$$

and $M_{t}^{i}:=N_{t}^{i}-\int_{0}^{t} \lambda_{s}^{i} \mathrm{~d} s$ is a $g_{t}$-martingale. Thus, by definition, we deduce that the jump times of the processes $N^{0}, N^{1}, N^{2}$, and $D$ are $g$-stopping times.

As in Tardelli [20], the intensities of $N^{0}, N^{1}$, and $N^{2}$ can take into account the arrival of news reaching the market that produces a sudden increase in the trading activity followed by a progressive normalization. By adequately choosing the form of these intensities, we are able to describe also deterministic features such as seasonalities. Note that the intensity of the jump times of $Y_{t}$ is assumed to be $g_{t}$-measurable in order to take into account the role of the unobserved process $X$. In this setting, the dynamics of $X$ and $Y$ can be given by assuming the following representation:

$$
\begin{gathered}
X_{t}:=\int_{0}^{t} \xi_{u}^{0} \mathrm{~d} N_{u}^{0}+\int_{0}^{t} \xi_{u}^{1} \mathrm{~d} N_{u}^{1}+\int_{0}^{t} \xi_{u}^{2} \mathrm{~d} N_{u}^{2}+\int_{0}^{t} \xi_{u}^{3} \mathrm{~d} D_{u}, \\
Y_{t}:=\int_{0}^{t} \eta_{u}^{1} \mathrm{~d} N_{u}^{1}-\int_{0}^{t} \eta_{u}^{2} \mathrm{~d} N_{u}^{2}+\int_{0}^{t} \beta_{u} \mathrm{~d} D_{u},
\end{gathered}
$$

where $\xi_{t}^{i}$ for $i=0,1,2,3$ are $g_{t}$-predictable stochastic processes and $\eta_{t}^{1}, \eta_{t}^{2}$, and $\beta_{t}$ are just $\mathcal{O}_{t}$-predictable. 
From a financial point of view, the processes $X$ and $Y$ are supposed to have some stochastic changes, due to market perturbations caused by the arrival of news. If at time $t$ news, not observable by the investor, reaches the market, only the process $N^{0}$ increases and $X$ has a change of size $\xi_{t}^{0}$. Otherwise, if at $t$ news, observable by the investor, reaches the market, we have a positive jump of the processes $N^{1}$ or $N^{2}$. In this last case, a change in $X$ happens of size $\xi^{1}$ or $\xi^{2}$, as well as a change in $Y$ of size $\eta^{1}$ or $\eta^{2}$. Note that only these last changes are observable. If a default occurs, $Y$ has a jump of size $\beta$, and $X$ of size $\xi^{3}$. Note that since $X$ is not observed and trading is stopped at $\tau$, we could not consider the jump of $X$ at the moment of the default, but this is a choice which does not simplify the procedure.

Moreover, for some real positive constant $\underline{\eta}$ and $\bar{\eta}$, let us assume that, for $i=1,2$,

$$
\underline{\eta} \leq \eta_{t}^{i} \leq \bar{\eta}
$$

In common with many authors in this field, let us assume that $\beta_{\tau}>-1$. According to the existing literature, see, e.g. Lim and Quenez [15], this condition is equivalent to $\beta_{t}>-1$ for any $0 \leq t \leq T$ almost surely (a.s.) and $S_{\tau}=S_{\tau-}\left(1+\beta_{\tau}\right)$, and the price process $S$ is still positive after the default $\tau$.

Remark 1. If the price process is strictly increasing or strictly decreasing, the model does not admit any equivalent martingale measure. The particular structure chosen for the dynamics of the process $Y$ is the simplest one allowing the existence of equivalent martingale measures; see, e.g. Tardelli [20] and the references therein.

As a consequence of this observation, we assume, as in Frittelli [9], that the set of the equivalent martingale measures is not empty, thus, this set is not necessarily a singleton and so we have a set of prices all compatible with the 'no arbitrage' condition. The financial interpretation of this approach is illustrated in Frittelli [9] as well as the relationship between the minimization of the relative entropy and the maximization of the exponential utility function.

Remark 2. The model presented in this section could be established in a more formal way. For example, as in Tardelli [20], we could introduce a Markovian structure which would allow us to obtain the process $(X, Y, D)$ as a solution of a martingale problem. Otherwise, inspired by Ceci [5], we could look at (3) and (4), as a system of stochastic differential equations and, by introducing some suitable assumptions, we could discuss its existence and uniqueness. However, the main part of this paper, which deals with the utility maximization property, does not depend on the structure of the model introduced.

As a conclusion to this section, by a standard application of the Itô formula, setting

$$
c_{u}:=\left(\mathrm{e}^{\eta_{u}^{1}}-1\right) \lambda_{u}^{1}+\left(\mathrm{e}^{-\eta_{u}^{2}}-1\right) \lambda_{u}^{2}+\left(\mathrm{e}^{\beta_{u}}-1\right) \lambda_{u}^{3},
$$

the representation of the price process as a $g_{t}$-semimartingale is given by

$$
S_{t}=S_{0}+\int_{0}^{t} S_{u} c_{u} \mathrm{~d} u+M_{t}^{S}
$$

where $M_{t}^{S}$ is a $g_{t}$-local martingale represented as

$$
M_{t}^{S}=\int_{0}^{t} S_{u-}\left(\mathrm{e}^{\eta_{u}^{1}}-1\right) \mathrm{d} M_{u}^{1}+\int_{0}^{t} S_{u-}\left(\mathrm{e}^{-\eta_{u}^{2}}-1\right) \mathrm{d} M_{u}^{2}+\int_{0}^{t} S_{u-}\left(\mathrm{e}^{\beta_{u}}-1\right) \mathrm{d} M_{u}^{3} .
$$




\section{The $\mathcal{O}$-representation}

Recall that this paper deals with the point of view of partially informed investors. The available information for these traders, in this economy at a certain time $t \in[0, T]$, are the price of the financial asset up to that time and the default indicating process.

To this end, first of all, the $\mathcal{O}_{t}$-predictable projection of the processes involved in this model has to be found.

Definition 1. Given a process $\Gamma_{t}, g_{t}$-adapted, let us denote by ${ }^{\mathrm{p}} \Gamma_{t}$ the predictable projection on $\mathcal{O}$ and by ${ }^{\circ} \Gamma_{t}$ the optional projection on $\mathcal{O}$. For each $\mathcal{O}_{t}$-predictable stopping time $\sigma$ (see Jacod [12, Theorem 1.23]), ${ }^{\mathrm{p}} \Gamma_{\sigma} \equiv \mathbb{E}\left[\Gamma_{\sigma} \mid \mathcal{O}_{\sigma-}\right]$ and, for each $\sigma, \mathcal{O}_{t}$-stopping time (see Ethier and Kurtz [7, Optional projection theorem, Theorem 4.2 p. 72]), ${ }^{\circ} \Gamma_{\sigma} \equiv \mathbb{E}\left[\Gamma_{\sigma} \mid \mathcal{O}_{\sigma}\right]$.

In the continuous framework, the option projection and the predictable projection coincide. This is not the case for discontinuous models. The situation is described by the following lemma, the proof of which follows that given in Frey [8] and Tardelli [20]. The proof is recalled here for the sake of completeness.

Lemma 1. If the process $\Gamma_{t}$ is locally integrable and with finite variation, the predictable and the optional projections satisfy ${ }^{\mathrm{o}} \Gamma_{t-}={ }^{\mathrm{p}} \Gamma_{t}$.

Proof. For each $\mathcal{O}_{t}$-predictable stopping time $\sigma$, since $\{\sigma<+\infty\} \in \mathcal{O}_{\sigma}$,

$$
\mathbb{E}\left[{ }^{\mathrm{p}} \Gamma_{\sigma} \mathbf{1}_{\{\sigma<+\infty\}}\right]=\mathbb{E}\left[\Gamma_{\sigma} \mathbf{1}_{\{\sigma<+\infty\}}\right]=\mathbb{E}\left[{ }^{\mathrm{o}} \Gamma_{\sigma} \mathbf{1}_{\{\sigma<+\infty\}}\right] .
$$

On the other hand, there exists a version of the process ${ }^{0} \Gamma_{t}$ with càdlàg trajectories and, consequently, ${ }^{o} \Gamma_{t-}$ is a $\left(\mathbb{P}, \mathcal{O}_{t}\right)$-predictable process. Moreover, the jump times of ${ }^{o} \Gamma_{t-}$, which coincide with the jump times of $Y_{t}$, are totally inaccessible. Hence, for each $\left(\mathbb{P}, \mathcal{O}_{t}\right)$-predictable stopping time ${ }^{\mathrm{o}} \Gamma_{\sigma-}={ }^{\mathrm{p}} \Gamma_{\sigma}$ (see, e.g. [6]), $\mathbb{E}\left[{ }^{\mathrm{o}} \Gamma_{\sigma-} \mathbf{1}_{\{\sigma<+\infty\}}\right]=\mathbb{E}\left[{ }^{\mathrm{p}} \Gamma_{\sigma} \mathbf{1}_{\{\sigma<+\infty\}}\right]$. The thesis is achieved by noting that a deterministic time $\sigma \equiv t$ is a $\left(\mathbb{P}, \mathcal{O}_{t}\right)$-predictable stopping time.

Introducing the filter, i.e. the probability measure-valued process $\pi_{t}$, which is the càdlàg version of the conditional expectation with respect to $\mathcal{O}_{t}$,

$$
{ }^{\mathrm{o}} \Gamma_{t}=\pi_{t}(\Gamma)=\mathbb{E}\left[\Gamma_{t} \mid \mathcal{O}_{t}\right], \quad{ }^{\mathrm{p}} \Gamma_{t}={ }^{\mathrm{o}} \Gamma_{t-}=\pi_{t-}(\Gamma),
$$

where, for any bounded measurable $f(t, x)$, recall that $\pi_{t}(f)=\mathbb{E}\left[f\left(t, X_{t}\right) \mid \mathcal{O}_{t}\right]$. As a consequence, in Proposition 1 below, the $\mathcal{O}_{t}$-semimartingale representation of the price process is deduced.

For notational convenience, let $m(\mathrm{~d} t, \mathrm{~d} \phi)$ be the integer-valued random measure associated to $Y_{t}$ (see Bremaud [3] and Jacod [12]), defined, for $t \in[0, T], \phi \in \mathbb{R}$, as

$$
m((0, t], \mathrm{d} \phi)=\sum_{s \leq t} \mathbf{1}_{\left\{Y_{s}-Y_{s-} \neq 0\right\}} \delta_{\left\{Y_{s}-Y_{s-}\right\}}(\mathrm{d} \phi) .
$$

Let us denote by $v_{t}(\mathrm{~d} \phi) \mathrm{d} t$ the dual $g_{t}$-predictable projection of $m$ and by $\hat{v}_{t}(\mathrm{~d} \phi) \mathrm{d} t$ the dual $\mathcal{O}_{t}$-predictable projection of $m$. In the following lemma the structures of $v_{t}(\mathrm{~d} \phi) \mathrm{d} t$ and of $\hat{v}_{t}(\mathrm{~d} \phi) \mathrm{d} t$ are given. This is a crucial link between the cases of partially informed and fully informed investors. 
Lemma 2. Since $\xi^{i}$ for $i=0,1,2,3$ are $g_{t}$-predictable stochastic processes, while $\eta^{1}, \eta^{2}$, and $\beta$ are $\mathcal{O}_{t}$-predictable,

$$
\begin{gathered}
v_{t}(\mathrm{~d} \phi)=\lambda_{t}^{1} \delta_{\left\{\eta_{t}^{1}\right\}}(\mathrm{d} \phi)+\lambda_{t}^{2} \delta_{\left\{-\eta_{t}^{2}\right\}}(\mathrm{d} \phi)+\lambda_{t}^{3} \delta_{\left\{\beta_{t}\right\}}(\mathrm{d} \phi) \\
\hat{v}_{t}(\mathrm{~d} \phi)=\pi_{t-}\left(v_{\cdot}(\mathrm{d} \phi)\right)=\pi_{t-}\left(\lambda^{1} \delta_{\left\{\eta_{.}\right\}}(\mathrm{d} \phi)+\lambda^{2} \delta_{\left\{-\eta^{2}\right\}}(\mathrm{d} \phi)+\lambda^{3} \delta_{\left\{\beta_{3}\right\}}(\mathrm{d} \phi)\right) .
\end{gathered}
$$

Proof. Setting $\Delta Y_{s}=Y_{s}-Y_{s-}, \Delta D_{s}=D_{s}-D_{s-}$, and $\Delta X_{s}=X_{s}-X_{s-}$, the integervalued random measure associated to $Y_{t}$ can be written as

$$
\begin{aligned}
m((0, t], \mathrm{d} \phi)=\sum_{s \leq t}[ & \delta_{\left\{\Delta Y_{s}\right\}}(\mathrm{d} \phi) \mathbf{1}_{\left\{\Delta Y_{s}>0\right\}}\left(1-\Delta D_{s}\right)+\delta_{\left\{\Delta Y_{s}\right\}}(\mathrm{d} \phi) \mathbf{1}_{\left\{\Delta Y_{s}<0\right\}}\left(1-\Delta D_{s}\right) \\
& \left.+\delta_{\left\{\Delta Y_{s}\right\}}(\mathrm{d} \phi) \Delta D_{s}\right] .
\end{aligned}
$$

Thus, as far as (7) and (8) are concerned, for any bounded positive $g_{t}$-predictable $f(t, \phi)$, the process $\int_{0}^{t} \int_{\mathbb{R}} f(s, \phi)\left(m(\mathrm{~d} s, \mathrm{~d} \phi)-v_{s}(\mathrm{~d} \phi) \mathrm{d} s\right)$ is a $g_{t}$-martingale.

Equation (8) is a consequence of Lemma 1, taking into account that, if $M_{t}$ is a $g_{t}$-martingale then $\pi_{t}(M)=\mathbb{E}\left[M_{t} \mid \mathcal{O}_{t}\right]$ is an $\mathcal{O}_{t}$-martingale. Hence, choosing a bounded positive $\mathcal{O}_{t^{-}}$ predictable $f(t, \phi), \mathbb{E}\left[\int_{0}^{t} \int_{\mathbb{R}} f(s, \phi)\left(m(\mathrm{~d} s, \mathrm{~d} \phi)-v_{s}(\mathrm{~d} \phi) \mathrm{d} s\right) \mid \mathcal{O}_{t}\right]$ is an $\mathcal{O}_{t}$-martingale. The thesis is achieved by proving that

$$
\tilde{M}_{t}=\mathbb{E}\left[\int_{0}^{t} \int_{\mathbb{R}} f(s, \phi) v_{s}(\mathrm{~d} \phi) \mathrm{d} s \mid \mathcal{O}_{t}\right]-\int_{0}^{t} \int_{\mathbb{R}} f(s, \phi) \hat{v}_{s}(\mathrm{~d} \phi) \mathrm{d} s
$$

is an $\mathcal{O}_{t}$-martingale. Indeed, for $t_{0}<t$,

$$
\begin{aligned}
\mathbb{E}\left[\tilde{M}_{t} \mid \mathcal{O}_{t_{0}}\right]= & \mathbb{E}\left[\int_{0}^{t_{0}} \int_{\mathbb{R}} f(s, \phi) v_{s}(\mathrm{~d} \phi) \mathrm{d} s \mid \mathcal{O}_{t_{0}}\right]+\mathbb{E}\left[\int_{t_{0}}^{t} \int_{\mathbb{R}} f(s, \phi) v_{s}(\mathrm{~d} \phi) \mathrm{d} s \mid \mathcal{O}_{t_{0}}\right] \\
& -\int_{0}^{t_{0}} \int_{\mathbb{R}} \mathbb{E}\left[f(s, \phi) v_{s}(\mathrm{~d} \phi) \mid \mathcal{O}_{s}\right] \mathrm{d} s \\
& -\int_{t_{0}}^{t} \int_{\mathbb{R}} \mathbb{E}\left[f(s, \phi) v_{s}(\mathrm{~d} \phi) \mid \mathcal{O}_{t_{0}}\right] \mathrm{d} s .
\end{aligned}
$$

Finally, we obtain $\mathbb{E}\left[\tilde{M}_{t} \mid \mathcal{O}_{t_{0}}\right]=\tilde{M}_{t_{0}}$ by noting that

$$
\mathbb{E}\left[\int_{t_{0}}^{t} \int_{\mathbb{R}} f(s, \phi) v_{s}(\mathrm{~d} \phi) \mathrm{d} s \mid \mathcal{O}_{t_{0}}\right]=\int_{t_{0}}^{t} \int_{\mathbb{R}} \mathbb{E}\left[f(s, \phi) v_{s}(\mathrm{~d} \phi) \mid \mathcal{O}_{t_{0}}\right] \mathrm{d} s .
$$

Proposition 1. The stock price process $S_{t}$ admits the following representation as an $\mathcal{O}_{t}$-semimartingale:

$$
S_{t}=S_{0}+\int_{0}^{t} S_{u-\pi_{u-}}(c) \mathrm{d} u+\bar{M}_{t}^{S},
$$

where $\pi_{u-}(c)=\pi_{u-}\left(\left(\mathrm{e}^{\eta^{1}}-1\right) \lambda^{1}+\left(\mathrm{e}^{-\eta^{2}}-1\right) \lambda^{2}+\left(\mathrm{e}^{\beta \cdot}-1\right) \lambda^{3}\right)$ and $\bar{M}_{t}^{S}$ is an $\mathcal{O}_{t}$-local martingale represented as

$$
\bar{M}_{t}^{S}=\int_{0}^{t} \int_{\mathbb{R}} S_{u-}\left(\mathrm{e}^{\phi}-1\right)\left(m(\mathrm{~d} u, \mathrm{~d} \phi)-\hat{v}_{u}(\mathrm{~d} \phi) \mathrm{d} u\right) .
$$


Proof. Taking into account that $S_{t}=S_{0}+\int_{0}^{t} \int_{\mathbb{R}} S_{u-}\left(\mathrm{e}^{\phi}-1\right) m(\mathrm{~d} u, \mathrm{~d} \phi)$ in order to obtain (9) and (10), recall the results of the previous lemma and note that

$$
\begin{aligned}
\int_{0}^{t} \int_{\mathbb{R}} S_{u-}\left(\mathrm{e}^{\phi}-1\right) \hat{v}_{u}(\mathrm{~d} \phi) \mathrm{d} u \\
\quad=\int_{0}^{t} S_{u-} \int_{\mathbb{R}} \pi_{u-}\left(\left(\mathrm{e}^{\phi}-1\right) \nu_{.}(\mathrm{d} \phi)\right) \mathrm{d} u \\
\quad=\int_{0}^{t} S_{u-} \pi_{u-}\left(\left(\mathrm{e}^{\eta^{1}}-1\right) \lambda^{1}+\left(\mathrm{e}^{-\eta^{2}}-1\right) \lambda^{2}+\left(\mathrm{e}^{\beta \cdot}-1\right) \lambda^{3}\right) \mathrm{d} u .
\end{aligned}
$$

The expression obtained in (8) for $\hat{v}_{t}(\mathrm{~d} \phi) \mathrm{d} t$ brings us to filtering techniques. The characterization of the filter will be given in Appendix A as the unique solution to the KushnerStratonovich equation.

\section{Hedging}

\subsection{The value process}

As a consequence of the previous discussions, the pair $\left(Y_{t}, D_{t}\right)$ become a totally observed marked point process, studied with respect to its internal filtration $\mathcal{O}_{t}$. As already observed in the introduction, this paper is devoted to the point of view of an investor, who can observe only the risky asset price and the default time. The investor can trade in a finite time window $[0, T]$ by investing in risky stocks and in a riskless bond, assuming also that there exists a default time on the market. The underlying traded asset is assumed to be a semimartingale driven by counting processes and the default indicating process.

As in Bielecki et al. [1], it is enough to formally define a generic defaultable European contingent claim with maturity $T$ through Definition 2 below.

Definition 2. On a suitable filtered probability space $(\Omega, \mathcal{F}, \mathbb{P})$, a defaultable claim maturing at $T$ is represented by a triplet $(Z, \tilde{Z}, \tau)$, where

(i) $\tau$ models the default time.

(ii) The promised payoff $Z$ represents the random payoff received by the owner of the claim maturing at $T$, provided that there is no default prior to $T$. This is an $\mathcal{O}_{T}$-measurable random variable such that $0 \leq Z \leq \bar{B}$ for a constant $\bar{B} \in \mathbb{R}^{+}$. The actual payoff at $T$ associated with $Z$ thus equals $Z \mathbf{1}_{\{T<\tau\}}$.

(iii) The $\mathcal{O}$-adapted recovery process $\tilde{Z}$ specifies the recovery payoff $\tilde{Z}_{\tau}$ received by the owner of a claim at the time of default (or at maturity), provided that the default occurred prior to or at the maturity date $T$ and such that $0 \leq \tilde{Z} \leq \bar{B}$.

Remark 3. In a typical contract, the payoff $Z$ is given by some function of the asset price and, thus, should be observed by the investor. This is the reason why, as in the existing literature, the payoff $Z$ is assumed to be an $\mathcal{O}_{T}$-measurable random variable and, analogously, the recovery process $\tilde{Z}$ is an $\mathcal{O}$-adapted recovery process.

In practice, hedging of a credit derivative after the default time is usually of minor interest. Also, in a model with a single default time, hedging after default reduces to replication of a nondefaultable claim; see, e.g. Tardelli [20].

In order to set a hedging problem, let us define $\Theta$ as the class of admissible strategies $\theta_{t}$, that are real-valued, $\mathcal{O}_{t}$-predictable, $S$-integrable, and self-financing. Recalling the definition 
of an integer-valued random measure associated to $\left(Y_{t}, D_{t}\right)$ given in (6), the wealth process is defined for $\theta \in \Theta$ as

$$
W_{t}^{\theta}=w_{0}+\int_{0}^{t} \theta_{r} \mathrm{~d} S_{r}=w_{0}+\int_{0}^{t} \int_{\mathbb{R}} \theta_{r} S_{r-}\left(\mathrm{e}^{\phi}-1\right) m(\mathrm{~d} r, \mathrm{~d} \phi) .
$$

Note that replication refers to the behavior of the wealth process $W_{t}^{\theta}$ on the random interval $[0, T \wedge \tau]$ only. Hence, for the purpose of replication of defaultable claims of the form $(Z, \tilde{Z}, \tau)$, it is sufficient to consider prices stopped at $T \wedge \tau$.

A perfect replication of a defaultable claim is described in Definition 3 below.

Definition 3. A self-financing strategy $\theta$ replicates a defaultable claim $(Z, \tilde{Z}, \tau)$ if $W_{t}^{\theta}$ satisfies the hedging conditions $W_{T}^{\theta} \mathbf{1}_{\{T<\tau\}}=Z \mathbf{1}_{\{T<\tau\}}$ and $W_{\tau}^{\theta} \mathbf{1}_{\{T \geq \tau\}}=\tilde{Z}_{\tau} \mathbf{1}_{\{T \geq \tau\}}$.

On the other hand, with the introduction of a pure jump process, the market becomes incomplete and a claim is not, in general, perfectly replicable; see, e.g. Runggaldier [18]. Thus, we have to use a hedging criterion under incompleteness. The choice in this paper consists of maximizing the expected utility of the wealth on a random time interval, i.e. to maximize $\mathbb{E}\left[u\left(W_{T \wedge \tau}^{\theta}-Z\left(1-D_{T}\right)-\tilde{Z}_{\tau} D_{T}\right)\right]$ for $\theta$ belonging to the class $\Theta$ of admissible strategies. Since $\mathbf{1}_{\{t \leq \tau\}}=1-D_{t-}$,

$$
W_{t \wedge \tau}^{\theta}=w_{0}+\int_{0}^{t} \int_{\mathbb{R}}\left(1-D_{r-}\right) \theta_{r} S_{r-}\left(\mathrm{e}^{\phi}-1\right) m(\mathrm{~d} r, \mathrm{~d} \phi) .
$$

After receiving the premium, the seller has to hedge in order to reduce the risk exposure. The expected utility of his final wealth gives him/her a measure of the quality of a self-financing strategy. At any $t \in[0, T]$, the agent invests the quantity $\theta_{t}$ in the risky traded asset. Thus, the investment process $\theta_{t}$ controls the dynamics of the wealth process. Hence, a stochastic control problem with only final reward arises.

Setting $w$ as the amount of capital at time $t$ and setting $\Theta_{t}$ as the set of the admissible strategies on the interval $[t, T]$, let us introduce the associated value process as

$$
\begin{aligned}
V_{t}(w) & =\operatorname{ess} \sup _{\theta \in \Theta_{t}} \mathbb{E}\left[u\left(W_{T \wedge \tau}^{\theta}-W_{t \wedge \tau}^{\theta}+w-Z\left(1-D_{T}\right)-\tilde{Z}_{\tau} D_{T}\right) \mid \mathcal{O}_{t}\right] \\
& =\operatorname{ess} \sup _{\theta \in \Theta_{t}} \mathbb{E}\left[u\left(w+\int_{t}^{T}\left(1-D_{r-}\right) \theta_{r} \mathrm{~d} S_{r}-Z\left(1-D_{T}\right)-\tilde{Z}_{\tau} D_{T}\right) \mid \mathcal{O}_{t}\right] .
\end{aligned}
$$

In particular, given the risk aversion parameter $\alpha \in \mathbb{R}^{+}$, let us consider an agent with exponential utility function $u_{a}(x)=1-\mathrm{e}^{-\alpha x}$.

Remark 4. Usually the strategies are required to make the associated wealth process positive. Note that this is a necessary assumption for power or logarithmic utility functions, which are defined on $\mathbb{R}^{+}$. But in the case of an exponential utility function which is finitely valued for all $x \in \mathbb{R}$, the wealth process is no longer required to be positive. However, from a financial point of view, it is natural to consider strategies such that at least any increment of the wealth is bounded from below; see, e.g. Schachermayer [19]. This last observation justifies Assumption 1 below.

Assumption 1. As in Lim and Quenez [15, Equation (4.1)], we assume that $\Theta$ consists of that process such that for any $\theta$ fixed and any $s \in[0, T]$, there exists a real constant $K_{s, \theta}$ such that $W_{t \wedge \tau}^{\theta}-W_{s \wedge \tau}^{\theta} \geq-K_{s, \theta}$ for $s \leq t \leq T$. 
Remark 5. As a consequence of Assumption $1, W^{\theta}$ is bounded from below and

$$
\mathbb{E}\left[\exp \left\{-\alpha \int_{t}^{T}\left(1-D_{r-}\right) \theta_{r} \mathrm{~d} S_{r}\right\}\right]<\infty
$$

Setting $B_{T}(\tau):=Z\left(1-D_{T}\right)+\tilde{Z}_{\tau} D_{T}$, then $B_{T}(\tau)$ is a function of $Z$, an $\mathcal{O}_{T}$-measurable random variable, and of $\tilde{Z}$., an $\mathcal{O}$-adapted process. Hence, the value process reduces to $V_{t}(w)=$ $1-\mathrm{e}^{-\alpha w} V_{t}$ and, since we are dealing with the partially observed case,

$$
V_{t}=\operatorname{ess} \inf _{\theta \in \Theta_{t}} \mathbb{E}\left[\exp \left\{-\alpha\left(\int_{t}^{T}\left(1-D_{r-}\right) \theta_{r} \mathrm{~d} S_{r}-B_{T}(\tau)\right)\right\} \mid \mathcal{O}_{t}\right] .
$$

The approach followed in this section is basically related to dynamic programming; see Tardelli [20]. As a consequence of this method, we characterize the process $V_{t}$ as the largest solution to a suitable BSDE. The properties of $V_{t}$ are given below and proved in Appendix B, since the proofs are just slight modifications of procedures performed in Lim and Quenez [15] and Tardelli [20].

Proposition 2. For $t \in[0, T \wedge \tau]$, the following statements hold true:

(i) the process $V_{t}$ is strictly positive and bounded and $V_{T}=\pi_{T}\left(\mathrm{e}^{\alpha B(\tau)}\right)$;

(ii) for any $\theta \in \Theta$, the process $V_{t} \exp \left\{-\alpha W_{t \wedge \tau}^{\theta}\right\}$ is an $\mathcal{O}_{t}$-submartingale;

(iii) $V_{t}$ is the largest $\mathcal{O}_{t}$-adapted process verifying (ii) such that $V_{T}=\pi_{T}\left(\mathrm{e}^{\alpha B .(\tau)}\right)$;

(iv) $\theta^{*} \in \Theta$ is an optimal strategy if and only if $V_{t} \exp \left\{-\alpha W_{t \wedge \tau}^{\theta^{*}}\right\}$ is an $\mathcal{O}_{t}$-martingale;

(v) the process $V_{t}$ admits an indistinguishable $\mathcal{O}_{t}$-adapted càdlàg representation.

\subsection{BSDEs: partial observation}

In this section we will prove that $V_{t}$ is the largest solution of a suitable BSDE. Note that we are not able to prove a uniqueness result for this BSDE and this justifies the discussion in the next section.

By Proposition 2, for a vanishing strategy, the process $V_{t}$ is a bounded and strictly positive $\mathcal{O}_{t}$-submartingale. Consequently, its Doob-Meyer decomposition is

$$
\mathrm{d} V_{t}=\mathrm{d} M_{t}^{V}+\mathrm{d} A_{t}^{V}
$$

where $M_{t}^{V}$ is a square-integrable martingale and $A_{t}^{V}$ is an increasing $\mathcal{O}_{t}$-predictable process such that $A_{0}^{V}=0$. Recalling (6) and by a classical representation of $M_{t}^{V}$,

$$
V_{t}=V_{0}+\int_{0}^{t} \int_{\mathbb{R}} R_{r}(\phi)\left[m(\mathrm{~d} r, \mathrm{~d} \phi)-\hat{v}_{r}(\mathrm{~d} \phi) \mathrm{d} r\right]+A_{t}^{V},
$$

where $R_{t}(\phi)$ is a $\phi$-indexed process $\mathcal{O}_{t}$-predictable, jointly measurable, and such that

$$
\mathbb{E}\left[\int_{0}^{t} \int_{\mathbb{R}} \pi_{r}\left(R_{\cdot}^{2}(\phi) \nu_{.}(\mathrm{d} \phi)\right) \mathrm{d} r\right]<+\infty
$$

The main contribution of this section is the next theorem. 
Theorem 1. For $t \in[0, T]$, the process $\left(V_{t}, R_{t}\right)$ verifies the following BSDE:

$$
\begin{aligned}
V_{t}= & \pi_{T}\left[\mathrm{e}^{\alpha B_{T}(\tau)}\right]-\int_{t}^{T} \int_{\mathbb{R}} R_{r}(\phi)\left[m(\mathrm{~d} r, \mathrm{~d} \phi)-\hat{v}_{r}(\mathrm{~d} \phi) \mathrm{d} r\right] \\
& + \text { ess } \inf _{\theta \in \Theta_{t}} \int_{t}^{T} \int_{\mathbb{R}}\left(V_{r-}+R_{r}(\phi)\right) K_{r}^{\theta}(\phi) \hat{v}_{r}(\mathrm{~d} \phi) \mathrm{d} r, \\
& K_{r}^{\theta}(\phi)=\exp \left\{-\alpha\left(1-D_{r-}\right) \theta_{r} S_{r-}\left(\mathrm{e}^{\phi}-1\right)\right\}-1 .
\end{aligned}
$$

Furthermore, $V_{t}$ is the largest solution to (13) and $R_{t}$ is uniquely determined by the martingale representation theorem.

Proof. By the definition of $W_{t}^{\theta}$, setting $C_{t}^{\theta}:=\exp \left(-\alpha\left(W_{t \wedge \tau}^{\theta}-w_{0}\right)\right)$, from Itô's formula it follows that $C_{t}^{\theta}=1+\int_{0}^{t} \int_{\mathbb{R}} C_{r-}^{\theta} K_{r}^{\theta}(\phi) m(\mathrm{~d} r, \mathrm{~d} \phi)$. Hence, from the product formula with (12), $C_{t}^{\theta} V_{t}=C_{0}^{\theta} V_{0}+\int_{0}^{t} C_{s-}^{\theta} \mathrm{d} V_{s}+\int_{0}^{t} V_{s-} \mathrm{d} C_{s}^{\theta}+\left[C^{\theta}, V\right]_{t}$, where $C_{0}^{\theta} V_{0}=1 V_{0}=V_{0}$ and

$$
\begin{gathered}
\int_{0}^{t} C_{s-}^{\theta} \mathrm{d} V_{s}=\int_{0}^{t} \int_{\mathbb{R}} C_{s-}^{\theta} R_{s}(\phi) m(\mathrm{~d} s, \mathrm{~d} \phi)-\int_{0}^{t} \int_{\mathbb{R}} C_{s-}^{\theta} R_{s-}(\phi) \hat{v}_{s}(\mathrm{~d} \phi) \mathrm{d} s+\int_{0}^{t} C_{s}^{\theta} \mathrm{d} A_{s}^{V}, \\
\int_{0}^{t} V_{s-} \mathrm{d} C_{s}^{\theta}=\int_{0}^{t} \int_{\mathbb{R}} V_{s-} C_{s-}^{\theta} K_{s}^{\theta}(\phi) m(\mathrm{~d} s, \mathrm{~d} \phi), \\
{\left[C^{\theta}, V\right]_{t}=\sum_{s \leq t} \Delta C_{s}^{\theta} \Delta V_{s}=\int_{0}^{t} \int_{\mathbb{R}} C_{s-}^{\theta} K_{s}^{\theta}(\phi) R_{s}(\phi) m(\mathrm{~d} s, \mathrm{~d} \phi) .}
\end{gathered}
$$

Therefore,

$$
\begin{aligned}
C_{t}^{\theta} V_{t}= & V_{0}+\int_{0}^{t} \int_{\mathbb{R}} C_{s-}^{\theta}\left[R_{s}(\phi)+\left(V_{s-}+R_{s}(\phi)\right) K_{s}^{\theta}(\phi)\right]\left[m(\mathrm{~d} s, \mathrm{~d} \phi)-\hat{v}_{s}(\mathrm{~d} \phi) \mathrm{d} s\right] \\
& +\int_{0}^{t} \int_{\mathbb{R}} C_{s-}^{\theta}\left(V_{s-}+R_{s}(\phi)\right) K_{s}^{\theta}(\phi) \hat{v}_{s}(\mathrm{~d} \phi) \mathrm{d} s+\int_{0}^{t} C_{s}^{\theta} \mathrm{d} A_{s}^{V} .
\end{aligned}
$$

Since $C_{t}^{\theta} V_{t}=\exp \left(-\alpha\left(W_{t \wedge \tau}^{\theta}-w_{0}\right)\right) V_{t}$ is an $\mathcal{O}_{t}$-submartingale, the bounded variation term has to be increasing for any strategy and it has to vanish for the optimal strategy, which implies that

$$
A_{t}^{V}=\int_{0}^{t} \int_{\mathbb{R}} R_{s}(\phi) \hat{v}_{s}(\mathrm{~d} \phi) \mathrm{d} s=-\operatorname{ess} \inf _{\theta \in \Theta_{t}} \int_{0}^{t} \int_{\mathbb{R}}\left(V_{s-}+R_{s}(\phi)\right) K_{s}^{\theta}(\phi) \hat{v}_{s}(\mathrm{~d} \phi) \mathrm{d} s .
$$

Finally, the last assertion is a consequence of Proposition 2 by noting that

$$
V_{t}=V_{T}-\int_{t}^{T} \mathrm{~d} M_{r}^{V}-\int_{t}^{T} A_{r}^{V} \mathrm{~d} r
$$

\section{A sequence converging to the value process}

Until now, the process $V_{t}$ was characterized as the largest solution to a suitable BSDE and results on the uniqueness of solutions that are not available, due to the fact that the generator does not have the Lipschitz property; see Carbone et al. [4]. Many authors, e.g. Bouchard and Elie [2], discuss methods to obtain a discrete-time approximation for the value function. Since they require existence and uniqueness for the solution of a BSDE, in this section a sequence $\left\{V_{t}^{k}\right\}_{k \in N}$ will be constructed such that

(i) $\left\{V_{t}^{k}\right\}_{k \in \mathbb{N}}$ is a nonincreasing sequence converging to $V_{t}$;

(ii) for $k \in \mathbb{N}, V_{t}^{k}$ is the unique solution of a BSDE with a Lipschitz generator. 
To achieve these results, we need a new assumption.

Assumption 2. The process $\beta_{t}, 0 \leq t \leq T$, is uniformly bounded.

Definition 4. Let us define, for a constant $C \in \mathbb{R}^{+}$and for any $k \in \mathbb{N}$,

$$
\Theta^{k}=\left\{\theta \in \Theta:\left|\theta_{u}\right| \leq k,\left|\theta_{u} S_{u-}\right| \leq C \text { for all } u \in[0, T] \text { a.s. }\right\} \subset \Theta
$$

and $\Theta_{t}^{k}$ is the set of strategies in $\Theta^{k}$ on the interval $[t, T \wedge \tau]$.

Let us define

$$
V_{t}^{k}=\mathrm{ess} \inf _{\theta \in \Theta_{t}^{k}} \pi_{t}\left[\exp \left\{-\alpha\left(\int_{t}^{T}\left(1-D_{r-}\right) \theta_{r} \mathrm{~d} S_{r}\right)\right\} \mathrm{e}^{\alpha B_{T}(\tau)}\right] .
$$

Note that the difference between $V_{t}^{k}$ and $V_{t}$, given in (11), relies only on the choice of the set of strategies. In spite of that, the results obtained previously still hold.

Remark 6. Recall that $\Theta^{k}$ is a bounded set and the argument of the expectation in (15) has finite mean value. Thus, Lebesgue's theorem applies and there exists an optimal strategy $\theta^{*, k} \in \Theta^{k}$.

As a consequence, all the results given in Proposition 2 hold for $V_{t}^{k}$, which, in particular, means that $V_{t}^{k}$ is a bounded and strictly positive $\mathcal{O}_{t}$-submartingale. Thus, its Doob-Meyer decomposition is $V_{t}^{k}=V_{0}^{k}+\int_{0}^{t} \mathrm{~d} M_{r}^{V^{k}}+\int_{0}^{t} \mathrm{~d} A_{r}^{V^{k}}$ with $M_{t}^{V^{k}}$ a square-integrable $\mathcal{O}_{t}$-martingale, $A_{t}^{V^{k}}$ increasing $\mathcal{O}_{t}$-predictable process such that $A_{0}^{V^{k}}=0$,

$$
\mathrm{d} M_{r}^{V^{k}}=\int_{\mathbb{R}} R_{r}^{k}(\phi)\left[m(\mathrm{~d} r, \mathrm{~d} \phi)-\hat{v}_{r}(\mathrm{~d} \phi) \mathrm{d} r\right], \quad \mathrm{d} A_{r}^{V^{k}}=\int_{\mathbb{R}} R_{r}^{k}(\phi) \hat{v}_{r}(\mathrm{~d} \phi) \mathrm{d} r,
$$

and where $R_{r}^{k}(\phi)$ are measurable $\mathcal{O}_{t}$-predictable processes such that

$$
\mathbb{E}\left[\int_{0}^{t} \int_{\mathbb{R}}\left(R_{r}^{k}(\phi)\right)^{2} \hat{v}_{r}(\mathrm{~d} \phi) \mathrm{d} r\right]<+\infty .
$$

For each $k \in \mathbb{N}, V_{t}^{k}$ is characterized as the unique solution of a BSDE.

Proposition 3. For $t \in[0, T]$ and $K_{r}^{\theta}(x)$ given in $(14),\left(V_{t}^{k}, R_{t}^{k}\right)$ is the unique positive càdlàg $\mathcal{O}_{t}$-adapted solution of the following BSDE with Lipschitz continuous generator:

$$
\begin{aligned}
V_{t}^{k}= & \pi_{T}\left[\mathrm{e}^{\alpha B_{T}(\tau)}\right]-\int_{t}^{T} \int_{\mathbb{R}} R_{r}^{k}(\phi)\left[m(\mathrm{~d} r, \mathrm{~d} \phi)-\hat{v}_{r}(\mathrm{~d} \phi) \mathrm{d} r\right] \\
& + \text { ess } \inf _{\theta \in \Theta_{t}^{k}} \int_{t}^{T} \int_{\mathbb{R}}\left(V_{r-}^{k}+R_{r}^{k}(\phi)\right) K_{r}^{\theta}(\phi) \hat{v}_{r}(\mathrm{~d} \phi) \mathrm{d} r .
\end{aligned}
$$

Proof. Taking into account Remark 6 and following the same approach as in Theorem 1, the process $\left(V_{t}^{k}, R_{t}^{k}\right)$ is a solution to (16). To obtain the uniqueness property, let

$$
f(t, v, r):=\mathrm{ess} \inf _{\theta \in \Theta_{t}^{k}}[v+r(\phi)] K_{t}^{\theta}(\phi) \hat{v}_{t}(\mathrm{~d} \phi) .
$$

Note that $\Theta^{k}$ is a bounded set by Definition 4 , the coefficients $\lambda^{1}, \lambda^{2}, \gamma$, and $K_{t}^{\theta}$ are bounded by (1), (2), (5), and Assumption 2. This implies that $f(t, v, r)$ is an infimum of linear terms with respect to $v$ and $r$ with uniformly bounded coefficients so that $f$ is a Lipschitz function with respect to $v$ and $r$. Thus, uniqueness follows by the results of Carbone et al. [4]. 
Theorem 2. Under Assumption 2, $V_{t}=\lim _{k \rightarrow+\infty} V_{t}^{k}$ a.s. for all $t \in[0, T]$.

The next proposition allow us to obtain the proof of this theorem.

Proposition 4. There exists a càdlàg $\mathcal{O}_{t}$-submartingale $\tilde{V}_{t}$ such that

$$
V_{t} \leq \lim _{k \rightarrow+\infty} V_{t}^{k}=\tilde{V}_{t} \quad \text { a.s. for all } t \in[0, T]
$$

Proof. For $t \in[0, T]$, by definition the strategies are such that $\Theta_{t}^{k} \subset \Theta_{t}$, hence, for all $k \in \mathbb{N}, V_{t}^{k} \geq V_{t}>0$ a.s. Moreover, $\Theta_{t}^{k} \subset \Theta_{t}^{k+1}$, which implies that $\left\{V_{t}^{k}\right\}_{k \in \mathbb{N}}$ is a nonincreasing sequence that is also lower bounded. Thus, there exists an adapted process

$$
\tilde{V}_{t}:=\lim _{k \rightarrow+\infty} \downarrow V_{t}^{k} \geq V_{t} \quad \text { a.s. for all } t \in[0, T],
$$

which is càdlàg by [6, Theorem VI.18].

Fix $0 \leq s \leq t \leq T$ and recall that, by Remark $6, V_{t}^{k}$ is an $\mathcal{O}_{t}$-submartingale, then for all $k \in \mathbb{N}, \mathbb{E}\left[V_{t}^{k} \mid \mathcal{O}_{s}\right] \geq V_{s}^{k} \geq \tilde{V}_{s} \geq V_{s}$ a.s. Hence, $\tilde{V}_{t}$ has finite mean value and by the monotone convergence theorem for conditional expectation, $\mathbb{E}\left[\tilde{V}_{t} \mid \mathcal{O}_{s}\right] \geq \tilde{V}_{s}$ a.s., which implies that the process $\tilde{V}$ is an $\mathcal{O}_{t}$-submartingale.

Proposition 5. For each $\theta \in \Theta$ bounded strategies, $\tilde{V}_{t} \exp \left(-\alpha W_{t \wedge \tau}^{\theta}\right)$ is an $\mathcal{O}_{t}$-submartingale. Moreover, the process $\theta^{*} \in \Theta$ bounded is an optimal strategy if and only if the process $\tilde{V}_{t} \exp \left(-\alpha W_{t \wedge \tau}^{\theta^{*}}\right)$ is an $\mathcal{O}_{t}$-martingale.

Proof. Since $\theta$ is bounded, there exists $n \in \mathbb{N}$ such that $\theta$ is uniformly bounded by $n$ and for each $k \geq n, \theta \in \Theta^{k}$. Thus, $V_{t}^{k} \exp \left(-\alpha W_{t \wedge \tau}^{\theta}\right)$ is an $\mathcal{O}_{t}$-submartingale. Then, by the monotone convergence theorem for conditional expectation, $\tilde{V}_{t} \exp \left(-\alpha W_{t \wedge \tau}^{\theta}\right)$ is an $\mathcal{O}_{t}$-submartingale. Furthermore, the Doob-Meyer decomposition of the càdlàg $\mathcal{O}$-submartingale $\tilde{V}$ is

$$
\tilde{V}_{t}=\tilde{V}_{0}+\int_{0}^{t} \mathrm{~d} \tilde{M}_{r}^{V}+\int_{0}^{t} \mathrm{~d} \tilde{A}_{r}^{V}
$$

with $\tilde{M}_{t}^{V}$ a square-integrable $\mathcal{O}_{t}$-martingale and $\tilde{A}_{t}^{V}$ an increasing $\mathcal{O}_{t}$-predictable process with $\tilde{A}_{0}^{V}=0$, and such that

$$
\mathrm{d} \tilde{M}_{r}^{V}=\int_{\mathbb{R}} \tilde{R}_{r}(\phi)\left[m(\mathrm{~d} r, \mathrm{~d} \phi)-\hat{v}_{r}(\mathrm{~d} \phi) \mathrm{d} r\right], \quad \mathrm{d} \tilde{A}_{r}^{V}=\int_{\mathbb{R}} \tilde{R}_{r}(\phi) \hat{v}_{r}(\mathrm{~d} \phi) .
$$

As in the proof of Theorem 1, by the product formula, $\tilde{V}_{t} \exp \left(-\alpha W_{t \wedge \tau}^{\theta}\right)=\tilde{M}_{t}^{\theta}+\tilde{A}_{t}^{\theta}$ with

$$
\begin{gathered}
\tilde{A}_{t}^{\theta}=\int_{0}^{t} \mathrm{e}^{-\alpha W_{r \wedge \tau}^{\theta}} \int_{\mathbb{R}}\left[\tilde{R}_{r}(\phi)+\left(\tilde{V}_{r}+\tilde{R}_{r}(\phi)\right) K_{r}^{\theta}(\phi)\right] \hat{v}_{r}(\mathrm{~d} \phi) \mathrm{d} r, \\
\tilde{M}_{t}^{\theta}=\int_{0}^{t} \mathrm{e}^{-\alpha W_{r \wedge \tau}^{\theta}} \int_{\mathbb{R}}\left[\tilde{R}_{r}(\phi)+\left(\tilde{V}_{r-}+\tilde{R}_{r}(\phi)\right) K_{r}^{\theta}(\phi)\right]\left[m(\mathrm{~d} r, \mathrm{~d} \phi)-\hat{v}_{r}(\mathrm{~d} \phi) \mathrm{d} r\right],
\end{gathered}
$$

where $\tilde{A}_{0}^{\theta}=0$ and $\tilde{M}_{0}^{\theta}=0$. Since, for each strategy $\theta \in \Theta$ bounded, $\tilde{V}_{t} \exp \left(-\alpha W_{t \wedge \tau}^{\theta}\right)$ is an $\mathcal{O}_{t}$-submartingale, then $\mathrm{d} \tilde{A}_{t}^{\theta} \geq 0$ a.s. Moreover, by the proof of Proposition 2(iv) (see Appendix B), it is a martingale for the optimal strategy, which means that

$$
\mathrm{d} \tilde{A}_{r}^{V}=\int_{\mathbb{R}} \tilde{R}_{r}(\phi) \hat{v}_{r}(\mathrm{~d} \phi)=-\operatorname{ess} \inf _{\theta \in \Theta_{r}^{k}} \int_{\mathbb{R}} \pi_{r}\left(\left(\tilde{V} .+\tilde{R}_{.}\right) K_{.}^{\theta}(\phi) \nu_{.}(\mathrm{d} \phi)\right) .
$$


Proposition 6. For each $t \in[0, T], V_{t} \geq \tilde{V}_{t}$ a.s.

Proof. For any $\theta \in \Theta$ and for each $k \in \mathbb{N}$, let $\theta_{t}^{k}:=\theta_{t} \mathbf{1}_{\{|\theta| \leq k\}}$. The strategy $\theta^{k}$ is uniformly bounded, but not necessarily admissible. To this end, let us define for each $(k, n) \in \mathbb{N}^{2}$ the stopping time $\tau_{k, n}:=\inf \left\{t \geq 0,\left|W_{t \wedge \tau}^{\theta^{k}}\right| \geq n\right\}$ and the strategy $\theta_{t}^{k, n}:=$ $\theta_{t}^{k} \mathbf{1}_{\left\{\tau_{k, n} \geq t\right\}}=\theta_{t} \mathbf{1}_{\{|\theta| \leq k\}} \mathbf{1}_{\tau_{k, n} \geq t}$. By construction, the strategy $\theta^{k, n} \in \Theta^{k}$ for each $(k, n)$ and $\theta_{t}=\lim _{k \rightarrow+\infty} \lim _{n \rightarrow+\infty} \theta_{t}^{k, n}$ a.s. Let $\widetilde{\Theta}$ be the set of all uniformly bounded admissible strategies, setting

$$
\tilde{Q}_{t}(\theta):=\int_{0}^{t} \int_{\mathbb{R}} \pi_{s}\left(\left(\tilde{V}_{.}+\tilde{R}_{.}\right) K_{.}^{\theta}(\phi) \nu_{.}(\mathrm{d} \phi)\right) \mathrm{d} s,
$$

then ess $\inf _{\theta \in \widetilde{\Theta}_{t}} \tilde{Q}_{t}(\theta)=\operatorname{ess}_{\inf _{\theta \in \Theta_{t}}} \tilde{Q}_{t}(\theta)$. Hence, $(\tilde{V}, \tilde{R})$ is a solution of the BSDE (13). By noting that $V_{t}$ is the largest process satisfying the same BSDE, $\tilde{V}_{t} \leq V_{t}$ a.s.

\section{Appendix A. Filtering}

Recall again that the filter $\pi_{t}$ is the probability measure-valued process, which is the càdlàg version of the conditional expectation, i.e. for any bounded measurable $F$,

$$
\pi_{t}(F)=\mathbb{E}\left[F_{t}\left(X_{t}\right) \mid \mathcal{O}_{t}\right] .
$$

The filter satisfies a stochastic differential equation known as the Kushner-Stratonovich equation, which we are going to write using the classical innovation method; see, e.g. [3].

To this end, note that, even if $Y(t)$ is not a counting process, the counting processes $N^{i}$, $i=0,1,2$ and $D$ do not have common jump times and, moreover,

$$
\mathcal{O}_{t}:=\sigma\left\{Y_{s}, D_{s}, 0 \leq s \leq t\right\} \equiv \sigma\left\{N_{s}^{1}, N_{s}^{2}, D_{s}, 0 \leq s \leq t\right\}
$$

Then our problem reduces to finding the filter, given $\mathcal{O}_{t}$. For notational convenience, from now on let $N_{t}^{3}:=D_{t}$. Taking into account the assumptions made in Section 2 on the model, for a real-valued function $F_{t}(x)$, bounded, and measurable with respect to $(t, x) \in \mathbb{R}^{+} \times \mathbb{R}$, and absolutely continuous with respect to $t$,

$$
F_{t}\left(X_{t}\right)=F_{0}\left(X_{0}\right)+\sum_{s \leq t} \sum_{j=0,1,2,3}\left[F_{s}\left(X_{s-}+\xi_{s}^{j}\right)-F_{s}\left(X_{s-}\right)\right] \Delta N_{s}^{j} .
$$

Hence, setting

$$
\begin{gathered}
\mathcal{A}_{s} F=\sum_{j=0,1,2,3}\left[F_{s}\left(X_{s-}+\xi_{s}^{j}\right)-F_{s}\left(X_{s-}\right)\right] \lambda_{s}^{j}, \\
F_{t}\left(X_{t}\right)=F_{0}\left(X_{0}\right)+\int_{0}^{t} \mathcal{A}_{s} F \mathrm{~d} s+\sum_{j=0,1,2,3} \int_{0}^{t}\left[F_{s}\left(X_{s-}+\xi_{s}^{j}\right)-F_{s}\left(X_{s-}\right)\right]\left(\mathrm{d} N_{s}^{j}-\lambda_{s}^{j} \mathrm{~d} s\right),
\end{gathered}
$$

the process $F_{t}\left(X_{t}\right)$ admits a $g_{t}$-semimartingale representation such that

$$
F_{t}\left(X_{t}\right)-F_{0}\left(X_{0}\right)-\int_{0}^{t}\left[\frac{\partial}{\partial s} F_{s}\left(X_{s}\right)+\mathcal{A}_{s} F\right] \mathrm{d} s
$$

is a $g_{t}$-martingale. In particular, for $f(x)$, real-valued, bounded, and measurable, $f\left(X_{t}\right)$ admits the $g_{t}$-semimartingale representation $f\left(X_{t}\right)=f\left(X_{0}\right)+\int_{0}^{t} \mathcal{A}_{s} f \mathrm{~d} s+m_{t}^{f}$, where $m_{t}^{f}$ is a zeromean $g_{t}$-martingale. 
The next proposition is a classical result; see [3, Theorem VIII-T9].

Proposition 7. For some $\mathcal{O}_{t}$-predictable processes $H_{t}^{j}$, the main tools needed in order to obtain the filtering equation are the following equations

$$
\begin{gathered}
\mathbb{E}\left[\int_{0}^{t} \Psi_{s}^{j, 1} H_{s}^{j} \pi_{s}\left(\lambda^{j}\right) \mathrm{d} s\right]=\mathbb{E}\left[\int_{0}^{t} f\left(X_{s}\right) H_{s}^{j} \lambda_{s}^{j} \mathrm{~d} s\right], \\
\mathbb{E}\left[\int_{0}^{t} \Psi_{s}^{j, 2} H_{s}^{j} \pi_{s}\left(\lambda^{j}\right) \mathrm{d} s\right]=\mathbb{E}\left[\int_{0}^{t} f\left(X_{S}\right) H_{s}^{j} \pi_{s}\left(\lambda^{j}\right) \mathrm{d} s\right], \\
\mathbb{E}\left[\int_{0}^{t} \Psi_{s}^{j, 3} H_{s}^{j} \pi_{s}\left(\lambda^{j}\right) \mathrm{d} s\right]=\mathbb{E}\left[\int_{0}^{t}\left[f\left(X_{S}\right)-f\left(X_{s-}\right)\right] H_{s}^{j} \mathrm{~d} N_{s}^{j}\right] .
\end{gathered}
$$

Theorem 3. The probability measure-valued process $\pi_{t}(f)$, which is the càdlàg version of $\mathbb{E}\left[f\left(X_{t}\right) \mid \mathcal{O}_{t}\right]$, is the solution to the Kushner-Stratonovich equation

$$
\begin{aligned}
& \pi_{t}(f)= f\left(X_{0}\right)+\int_{0}^{t} \pi_{s}(\mathcal{A} . f) \mathrm{d} s \\
&+\sum_{j=1}^{3} \int_{0}^{t}\left(\pi_{s-}\left(\lambda^{j}\right)\right)^{+}\left\{\pi_{s-}\left(\lambda^{j} f\right)-\pi_{s-}\left(\lambda^{j}\right) \pi_{s-}(f)+\pi_{s-}\left(R_{.}^{j} f\right)\right\} \\
& \quad \times\left(\mathrm{d} N_{s}^{j}-\pi_{s-}\left(\lambda^{j}\right) \mathrm{d} s\right),
\end{aligned}
$$

where $R_{s}^{j} f=\left[f\left(X_{s-}+\xi_{s}^{j}\right)-f\left(X_{s-}\right)\right] \lambda_{s}^{j}$ for $j=1,2,3$ and, taking into account that $\lambda^{3}$ can vanish, $a^{+}=(1 / a) \mathbf{1}_{\{a>0\}}$, as usual in the filtering theory.

Proof. By [3, Theorem VIII-T9], the $\mathcal{O}_{t}$-semimartingale representation for the filter is

$$
\pi_{t}(f)=f\left(X_{0}\right)+\int_{0}^{t} \pi_{s}(\mathcal{A} . f) \mathrm{d} s+M_{t}^{f}
$$

where $M_{t}^{f}$ is an $\mathcal{O}_{t}$-martingale. Therefore, there exist $\mathcal{O}_{t}$-predictable processes $K_{t}^{j}$ such that

$$
M_{t}^{f}=\sum_{j=1}^{3} \int_{0}^{t} K_{s}^{j}\left(\mathrm{~d} N_{s}^{j}-\pi_{s-}\left(\lambda^{j}\right) \mathrm{d} s\right), \quad K_{s}^{j}=\Psi_{s}^{j, 1}-\Psi_{s}^{j, 2}+\Psi_{s}^{j, 3} .
$$

By (17b), $\Psi_{s}^{j, 2}=\pi_{s-}(f)$. For $j=1,2,3, i=1,3$, it follows that $\Psi_{s}^{j, i}$ are $\mathcal{O}_{t}$-predictable processes, which are uniquely determined for any nonnegative $\mathcal{O}_{t}$-predictable process $H_{s}^{j}$ by (17a) and (17c).

Thus, from the right-hand side of (17a), we have

$$
\mathbb{E}\left[\int_{0}^{t} H_{s}^{j} \mathbb{E}\left[f\left(X_{s}\right) \lambda_{s}^{j} \mid \mathcal{O}_{s}\right] \mathrm{d} s\right]=\mathbb{E}\left[\int_{0}^{t} H_{s}^{j} \pi_{s}\left(\lambda^{j} f\right) \mathrm{d} s\right] .
$$

This implies that $\Psi_{s}^{j, 1}$ coincides with $\left(\pi_{s-}\left(\lambda^{j}\right)\right)^{+} \pi_{s-}\left(\lambda^{j} f\right)$. As far as $\Psi_{s}^{j, 3}$ is concerned, since

$$
f\left(X_{s}\right)-f\left(X_{s-}\right)=\sum_{n=0,1,2,3}\left[f\left(X_{s-}+\xi_{s}^{n}\right)-f\left(X_{s-}\right)\right] \mathrm{d} N_{s}^{n},
$$


the right-hand side of (17c) becomes, for $j=1,2,3$,

$$
\mathbb{E}\left[\int_{0}^{t}\left[f\left(X_{s-}+\xi_{s}^{j}\right)-f\left(X_{s-}\right)\right] H_{s}^{j} \mathrm{~d} N_{s}^{j}\right]=\mathbb{E}\left[\int_{0}^{t} H_{s}^{j} \mathbb{E}\left[R_{s}^{j} f \mid \mathcal{O}_{s}\right] \mathrm{d} s\right] .
$$

Hence, $\Psi_{s}^{j, 3}$ coincides with $\left(\pi_{s-}\left(\lambda^{j}\right)\right)^{+} \pi_{s}\left(R_{.}^{j} f\right)$, which completes the proof.

Proposition 8. If $\pi$ and $\pi^{\prime}$ are two probability measure-valued càdlàg processes satisfying (18), then $\pi$ and $\pi^{\prime}$ coincide pathwise.

Proof. Since $\pi$ and $\pi^{\prime}$ satisfy (18), for a suitable positive constant $C$,

$$
\left|\pi_{t}(f)-\pi_{t}^{\prime}(f)\right| \leq C \Lambda\|f\|\left\{\int_{0}^{t}\left\|\pi_{s}-\pi_{s}^{\prime}\right\| \mathrm{d} s+\sum_{j=1}^{3} \int_{0}^{t}\left(\pi_{s-}\left(\lambda^{j}\right)\right)^{+}\left\|\pi_{s-}-\pi_{s-}^{\prime}\right\| \mathrm{d} N_{s}^{j}\right\} .
$$

Recalling that $\pi_{s-}\left(\lambda^{j}\right)$ is the predictable intensity of $N_{s}^{j}$,

$$
\mathbb{E}\left[\int_{0}^{t}\left(\pi_{s-}\left(\lambda^{j}\right)\right)^{+}\left\|\pi_{s-}-\pi_{s-}^{\prime}\right\| \mathrm{d} N_{s}^{j}\right] \leq \mathbb{E}\left[\int_{0}^{t}\left\|\pi_{s-}-\pi_{s-}^{\prime}\right\| \mathrm{d} s\right]
$$

for another suitable constant $C^{\prime}$, we have $\mathbb{E}\left[\left\|\pi_{t}-\pi_{t}^{\prime}\right\|\right] \leq C^{\prime} \int_{0}^{t} \mathbb{E}\left[\left\|\pi_{s}-\pi_{s}^{\prime}\right\|\right] \mathrm{d} s$. Thus, by Grönwall's inequality, $\mathbb{E}\left[\left\|\pi_{t}-\pi_{t}^{\prime}\right\|\right]=0$ for all $t>0$. Taking into account that for all positive $h \in \mathbb{Q}$,

$$
\mathbb{P}\left(\left\|\pi_{t}-\pi_{t}^{\prime}\right\|>\frac{1}{h}\right) \leq h \mathbb{E}\left[\left\|\pi_{t}-\pi_{t}^{\prime}\right\|\right]=0,
$$

and that the sequence $\left\{\left\|\pi_{t}-\pi_{t}^{\prime}\right\|>1 / h\right\}$ is nondecreasing when $h$ increases,

$$
\mathbb{P}\left(\cup_{h}\left\{\left\|\pi_{t}-\pi_{t}^{\prime}\right\|>\frac{1}{h}\right\}\right)=\lim _{h \rightarrow+\infty} \mathbb{P}\left(\left\|\pi_{t}-\pi_{t}^{\prime}\right\|>\frac{1}{h}\right)=0 .
$$

This, in turn, implies that for all $t>0, \mathbb{P}\left(\left\|\pi_{t}-\pi_{t}^{\prime}\right\|>0\right)=0$. Since $\left\|\pi_{t}-\pi_{t}^{\prime}\right\|$ has càdlàg trajectories, in any countable $\mathcal{T}$, dense subset of $(0, T)$,

$$
\mathbb{P}\left(\sup _{0 \leq t \leq T}\left\|\pi_{t}-\pi_{t}^{\prime}\right\|>0\right)=\mathbb{P}\left(\sup _{\mathcal{T}}\left\|\pi_{t}-\pi_{t}^{\prime}\right\|>0\right) \leq \sum_{t \in \mathcal{T}} \mathbb{P}\left(\left\|\pi_{t}-\pi_{t}^{\prime}\right\|>0\right)=0 .
$$

Hence, each solution for the filtering equation coincides pathwise with the filter.

\section{Appendix B. Proof of Proposition 2}

Proof of Proposition 2(i). Noting that the strategy $\theta \equiv 0$ belongs to $\Theta$, it follows that

$$
V_{t} \leq \pi_{T}\left(\mathrm{e}^{\alpha B_{.}(\tau)}\right)=\pi_{T}\left(\exp \left\{\alpha\left(Z\left(1-D_{.}\right)+\tilde{Z}_{\tau} D_{.}\right)\right\}\right) \leq \mathrm{e}^{\alpha \bar{B}} .
$$

Furthermore, by [19, Theorem 2.2], there exists an optimal strategy $\theta^{*} \in \Theta$. As a consequence

$$
\mathrm{e}^{\alpha \bar{B}} \geq V_{t}=\pi_{t}\left[\exp \left\{-\alpha \int_{t}^{T}\left(1-D_{r-}\right) \theta_{r}^{*} \mathrm{~d} S_{r}\right\} \mathrm{e}^{\alpha B_{T}(\tau)}\right]>0 .
$$


Proof of Proposition 2(ii). For any admissible strategy $\theta \in \Theta$, since, by the previous proposition, $V_{t}$ is strictly positive and bounded, it follows that $\alpha \in \mathbb{R}^{+}$and the wealth process is assumed bounded from below. Recalling Remark 5, $\mathbb{E}\left[V_{t} \exp \left\{-\alpha W_{t \wedge \tau}^{\theta}\right\}\right]<+\infty$, which, obviously, means that

$$
\mathbb{E}\left[\mathbb{E}\left[V_{t} \exp \left\{-\alpha W_{t \wedge \tau}^{\theta}\right\} \mid \mathcal{O}_{T}\right]\right]<+\infty .
$$

For $s \wedge \tau \leq t \wedge \tau \leq T \wedge \tau$,

$$
\begin{aligned}
\mathbb{E}\left[\exp \left(-\alpha\left(W_{t \wedge \tau}^{\theta}-W_{s \wedge \tau}^{\theta}\right)\right) V_{t} \mid \mathcal{O}_{s}\right] \\
\quad=\mathbb{E}\left[\exp \left\{-\alpha\left(\int_{s}^{t}\left(1-D_{r-}\right) \theta_{r} \mathrm{~d} S_{r}+\int_{t}^{T}\left(1-D_{r-}\right) \theta_{r}^{*} \mathrm{~d} S_{r}-B_{T}(\tau)\right)\right\} \mid \mathcal{O}_{s}\right] \\
\quad=\mathbb{E}\left[\exp \left\{-\alpha\left(\int_{s}^{T}\left(1-D_{r-}\right) \tilde{\theta}_{r} \mathrm{~d} S_{r}-B_{T}(\tau)\right)\right\} \mid \mathcal{O}_{s}\right],
\end{aligned}
$$

where $\tilde{\theta}_{u}$ is a strategy belonging to $\Theta$ defined by fixing $\theta$ and $\theta^{*}$ at time $t$. Hence,

$$
\mathbb{E}\left[\mathrm{e}^{-\alpha\left(W_{t \wedge \tau}^{\theta}-W_{s \wedge \tau}^{\theta}\right)} V_{t} \mid \mathcal{O}_{s}\right] \geq V_{s}
$$

and Proposition 2(ii) is proved.

Proof of Proposition 2(iii). Next, let $\tilde{V}_{t}$ be another process $\mathcal{O}_{t}$-adapted verifying Proposition 2(ii) and such that $\tilde{V}_{T}=\mathbb{E}\left[\mathrm{e}^{\alpha B_{T}(\tau)} \mid \mathcal{O}_{T}\right]$. Since $\tilde{V}_{t} \exp \left\{-\alpha W_{t \wedge \tau}^{\theta}\right\}$ is an $\mathcal{O}_{t}$-submartingale, successively

$$
\begin{gathered}
\mathbb{E}\left[\tilde{V}_{T} \exp \left\{-\alpha W_{T \wedge \tau}^{\theta}\right\} \mid \mathcal{O}_{t}\right] \geq \tilde{V}_{t} \exp \left\{-\alpha W_{t \wedge \tau}^{\theta}\right\}, \\
\mathbb{E}\left[\exp \left(\alpha B_{T}(\tau)\right) \exp \left(-\alpha\left(W_{T \wedge \tau}^{\theta}-W_{t \wedge \tau}^{\theta}\right)\right) \mid \mathcal{O}_{t}\right] \geq \tilde{V}_{t}
\end{gathered}
$$

and, finally,

$$
V_{t}=\operatorname{ess} \inf _{\theta \in \Theta_{t}} \mathbb{E}\left[\mathrm{e}^{\alpha B_{T}(\tau)} \mathrm{e}^{-\alpha\left(W_{T \wedge \tau}^{\theta}-W_{t \wedge \tau}^{\theta}\right)} \mid \mathcal{O}_{t}\right] \geq \tilde{V}_{t}
$$

Proof of Proposition 2(iv). If $\theta_{t}^{*}$ is an optimal strategy then

$$
V_{0}=\mathbb{E}\left[\exp \left\{-\alpha\left(W_{T \wedge \tau}^{\theta^{*}}-B_{T}(\tau)\right)\right\}\right]
$$

By the submartingale property $\mathbb{E}\left[V_{T} \exp \left\{-\alpha W_{T \wedge \tau}^{\theta^{*}}\right\} \mid \mathcal{O}_{t}\right] \geq V_{t} \exp \left\{-\alpha W_{t \wedge \tau}^{\theta^{*}}\right\}$ and taking the mean value of both sides, we obtain

$$
\mathbb{E}\left[V_{T} \exp \left\{-\alpha W_{T \wedge \tau}^{\theta^{*}}\right\}\right] \geq \mathbb{E}\left[V_{t} \exp \left\{-\alpha W_{t \wedge \tau}^{\theta^{*}}\right\}\right],
$$

which implies the desired martingale property. Indeed, since $W_{0 \wedge \tau}^{\theta^{*}}=0$,

$$
\mathbb{E}\left[V_{T} \exp \left\{-\alpha W_{T \wedge \tau}^{\theta^{*}}\right\}\right] \equiv \mathbb{E}\left[V_{0} \exp \left(-\alpha W_{0 \wedge \tau}^{\theta^{*}}\right)\right]
$$

Next, assuming that there exists a $\theta^{*}$ such that $V_{t} \exp \left\{-\alpha W_{t \wedge \tau}^{\theta^{*}}\right\}$ is an $\mathcal{O}_{t}$-martingale, namely, $V_{0}=\mathbb{E}\left[\exp \left\{-\alpha\left(W_{T}^{\theta^{*}}-B_{T}(\tau)\right)\right\}\right]$. At the same time we know that

$$
V_{0}=\operatorname{ess} \inf _{\theta \in \Theta} \mathbb{E}\left[\exp \left\{-\alpha\left(W_{T}^{\theta}-B_{T}(\tau)\right)\right\}\right]
$$

which means that $\theta_{t}^{*}$ is an optimal strategy. 
Proof of Proposition 2(v). Since $V_{t}$ is a submartingale, by Karatzas and Shreve [14], setting $\mathbb{Q}$ as the set of rational numbers, for any $t \in[0, T)$, there exist the limits

$$
\lim _{s \in[0, T] \cap \mathbb{Q}, s \downarrow t} V_{s}=V_{t^{+}}, \quad \lim _{s \in[0, T] \cap \mathbb{Q}, s \uparrow t} V_{s}=V_{t^{-}} .
$$

So, $V_{t^{+}}$for each $t \in[0, T]$ is well defined by setting

$$
V_{t^{+}}:=\lim _{s \in[0, T] \cap \mathbb{Q}, s \downarrow t} V_{s}, \quad 0 \leq t<T \quad \text { and } \quad V_{T^{+}}(\omega):=V_{T} .
$$

From the right-continuity of $\mathcal{O}_{t}, V_{t^{+}}$is $\mathcal{O}$-adapted. Furthermore, $V_{t^{+}}$and, for $\theta \in \Theta$, $\mathrm{e}^{-\alpha W_{t \wedge \tau}^{\theta}} V_{t^{+}}$are $\mathcal{O}$-submartingale. Indeed, for $s \leq t$ and for any sequence of rationals $\left\{t_{n}\right\}_{n \geq 1}$ converging down to $t$,

$$
\mathbb{E}\left[\mathrm{e}^{-\alpha W_{t_{n} \wedge \tau}^{\theta}} V_{t_{n}} \mid \mathcal{O}_{s}\right] \geq \mathrm{e}^{-\alpha W_{s \wedge \tau}^{\theta}} V_{s},
$$

and by the Lebesgue theorem for conditional expectation for $n \rightarrow+\infty$,

$$
\mathbb{E}\left[\mathrm{e}^{-\alpha W_{t \wedge \tau}^{\theta}} V_{t^{+}} \mid \mathcal{O}_{s}\right] \geq \mathrm{e}^{-\alpha W_{s \wedge \tau}^{\theta}} V_{s} .
$$

Again, for any sequence of rationals $\left\{s_{n}\right\}_{n \geq 1}$ converging down to $s$,

$$
\mathbb{E}\left[\mathrm{e}^{-\alpha W_{t \wedge \tau}^{\theta}} V_{t^{+}} \mid \mathcal{O}_{s_{n}}\right] \geq \mathrm{e}^{-\alpha W_{s_{n} \wedge \tau}^{\theta}} V_{s_{n}},
$$

and by the Lebesgue theorem for conditional expectation for $n \rightarrow+\infty$, by the right-continuity of $\mathcal{O}$,

$$
\mathbb{E}\left[\mathrm{e}^{-\alpha W_{t \wedge \tau}^{\theta}} V_{t^{+}} \mid \mathcal{O}_{s}\right] \geq \mathrm{e}^{-\alpha W_{s \wedge \tau}^{\theta}} V_{s^{+}},
$$

which gives the submartingale property of the process $\mathrm{e}^{-\alpha W_{t \wedge \tau}^{\theta}} V_{t^{+}}$. Choosing $\theta=0$ for $s=t$ and using the right-continuity of $\mathcal{O}$, it follows that $V_{t^{+}}=\mathbb{E}\left[V_{t^{+}} \mid \mathcal{O}_{t}\right] \geq V_{t}$ a.s.

On the other hand, since $V_{t}$ is the largest process $\mathcal{O}$-adapted, verifying Proposition 2(i) and for each $\theta \in \Theta$, the process $\mathrm{e}^{-\alpha W_{t \wedge \tau}^{\theta}} V_{t^{+}}$is an $\mathcal{O}$-submartingale, then for each $t \in[0, T]$, $V_{t^{+}} \leq V_{t}$ a.s., which implies that $V_{t^{+}}=V_{t}$ a.s.

\section{Acknowledgement}

The author is very grateful to the anonymous referees for the valuable comments and suggestions which helped to improve this paper.

\section{References}

[1] Bielecki, T. R., Jeanblanc, M. and Rutkowski, M. (2006). Hedging of credit derivatives in models with totally unexpected default. In Stochastic Processes and Applications to Mathematical Finance, J. Akahori et al. (eds), World Scientific, Singapore, 35-100

[2] Bouchard, B. AND Elie, R. (2008). Discrete-time approximation of decoupled forward-backward SDE with jumps. Stoch. Process. Appl. 118, 53-75.

[3] Brémaud, P. (1981). Point Processes and Queues. Martingale Dynamics. Springer, New York.

[4] Carbone, R., Ferrario, B. and Santacroce, M. (2008). Backward stochastic differential equations driven by càdlàg martingales. Theory Prob. Appl. 52, 304-314.

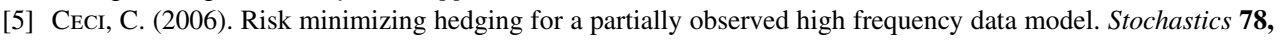
13-31.

[6] Dellacherie, C. And Meyer, P.-A. (1982). Probabilities and Potential. B. Theory of Martingales. NorthHolland, Amsterdam. 
[7] Ethier, S. N. AND Kurtz, T. G. (1986). Markov Processes: Characterization and Convergence. John Wiley, New York.

[8] Frey, R. (2000). Risk minimization with incomplete information in a model for high-frequency data. Math. Finance 10, 215-225.

[9] Frittelli, M. (2000). The minimal entropy martingale measure and the valuation problem in incomplete markets. Math. Finance 10, 39-52.

[10] Gerardi, A. ANd Tardelli, P. (2010). Stochastic control methods: hedging in a market described by pure jump processes. Acta Appl. Math. 111, 233-255.

[11] Hu, Y., Imkeller, P. And Müller, M. (2005). Utility maximization in incomplete markets. Ann. Appl. Prob. 15, 1691-1712.

[12] JACOD, J. (1979). Calcul Stochastique et Problèmes de Martingales (Lecture Notes Math. 714). Springer, Berlin.

[13] Jeanblanc, M., Yor, M. And Chesney, M. (2009). Mathematical Methods for Financial Markets. Springer, London.

[14] Karatzas, I. And Shreve, S. E. (1988). Brownian Motion and Stochastic Calculus. Springer, New York.

[15] Lim, T. AND Quenez, M.-C. (2011). Exponential utility maximization in an incomplete market with defaults. Electron. J. Prob. 16, 1434-1464.

[16] Mania, M. And Schweizer, M. (2005). Dynamic exponential utility indifference valuation. Ann. Appl. Prob. 15, 2113-2143.

[17] Prigent, J.-L. (2001). Option pricing with a general marked point process. Math. Operat. Res. 26, 50-66.

[18] Runggaldier, W. J. (2003). Jump-diffusion models. In Handbook of Heavy Tailed Distributions in Finance, Elsevier, Amsterdam, pp. 169-209.

[19] Schachermayer, W. (2001). Optimal investment in incomplete markets when wealth may become negative. Ann. Appl. Prob. 11, 694-734.

[20] Tardelli, P. (2011). Utility maximization in a pure jump model with partial observation. Prob. Eng. Inf. Sci. 25, 29-54.

[21] Tardelli, P. (2012). Modeling an incomplete market with default. In Proceedings of the 2nd International Conference on Stochastic Modelling and Simulation, Hikey Media, Royapettah, pp. 54-65. ISBN: 978-81-925286-4-9.

[22] Zariphopoulou, T. (2001). A solution approach to valuation with unhedgeable risks. Finance Stoch. 5, 61-82. 